\title{
Research on the Influence Mechanism of the Across-Industrial-Chain Investment Speed on Innovation Performance of AI Enterprises: Improvement Path of Artificial Intelligence Technology Application
}

\author{
Yan Chen, ${ }^{1}$ Fan Si, ${ }^{1}$ Xiying $\mathrm{Lu},{ }^{1}$ and $\mathrm{Xin} \mathrm{Li} \mathbb{C}^{2}$ \\ ${ }^{1}$ School of Economics and Management, Beijing University of Posts and Telecommunications, Beijing, China \\ ${ }^{2}$ School of Economics and Management, Civil Aviation University of China, Tianjin, China \\ Correspondence should be addressed to Xin Li; 2019071091@cauc.edu.cn
}

Received 30 September 2021; Revised 22 October 2021; Accepted 12 November 2021; Published 29 November 2021

Academic Editor: Sang-Bing Tsai

Copyright @ 2021 Yan Chen et al. This is an open access article distributed under the Creative Commons Attribution License, which permits unrestricted use, distribution, and reproduction in any medium, provided the original work is properly cited.

\begin{abstract}
This paper presents a regression analysis by using the system generalized method of moments (SYS-GMM) model as the main regression model and combining it with the fixed effect of panel data and acquires the basic empirical research data from Wind database. The research shows that the speed of cross-industrial-chain investment can improve the innovation ability of AI enterprises, and AI enterprises with deep technology accumulation can improve their innovation performance in the rapid across-industrial-chain investment. In this paper, an across-industrial-chain investment decision path model for AI enterprises is proposed for the first time, suggesting that AI enterprises should pay attention to the related factors of industry and AI enterprises when making across-industrial-chain investment decisions. This helps to express the determination of investment, integration, and reconstruction to the target AI enterprises, and it can also facilitate fast across-industrial-chain investment and improve the innovation performance of AI enterprises.
\end{abstract}

\section{Introduction}

Amid China's continuously advancing economic transformation and gradually deepening supply-side structural reform, more Chinese AI enterprises begin to adopt the horizontal integration strategies.

Existing literature pays more attention to the resource characteristics and differences among AI enterprises [1] and to the influence of industrial environment [2] and institutional environment [3] on the expansion of AI enterprises.

However, some researchers pointed out that it was difficult to truly reflect the dynamic and complex business reality faced by AI enterprises [4] from the static perspective alone. Therefore, it is essential to pay attention to the temporal characteristics of the expansion behavior.

In recent years, the rise of the temporal view of management provides a new perspective and research approach for studying the factors affecting the expansion performance of AI enterprises [5-7]. The temporal view of management takes the concept of time as the core and pays attention to how the characteristics of AI enterprises in the temporal dimension affect the generation, implementation, and transformation of strategies [8]. The temporal view of management introduces the dynamic research paradigm into the research of AI enterprise expansion, which is divided into six key modules: stage, opportunity, speed, duration, sequence, and frequency. Among them, speed, as one of the key symbols of time, has attracted wide attention from academic circles.

\section{Literature Review}

An agreement on how the expansion speed of AI enterprises affects their performance has not been reached in previous studies. Some studies believe that rapid expansion hinders the cultivation of learning capabilities of AI enterprises [9]. 
AI enterprises cannot fully enjoy the expansion results during rapid expansion, which may result in the diseconomy of time compression [10]. Some studies have also suggested that rapid expansion is conducive to enhancing the flexibility and environmental adaptability of AI enterprises, helping them fully grasp the opportunities and take the lead in market competition [4].

There are three reasons for the contradiction in the existing research conclusions. First of all, the definition of AI enterprise expansion is different under different research topics. Second, analysis of the boundary of the expansion of AI enterprises is still absent; that is, there is no distinction between the expansion directions of AI enterprises. Third, the internal mechanism between the expansion speed and the performance of AI enterprises has not been discussed in depth $[5,6,11]$.

To make up for the shortcomings of existing research, this paper introduces the concept of direction into the temporal view of management, taking the innovation ability that significantly affects the expansion performance of AI enterprises as the result variable to explore how AI enterprises' investment speed across production chains will affect their innovation performance.

\section{Research Model and Hypotheses}

AI enterprises can improve their innovation performance in the process of across-industrial-chain investment. At the market level, AI enterprises obtain the key resources needed for production and operation through rapid investment to support the development of their own innovation capability. It is found that rapid expansion can help AI enterprises accelerate their learning behavior, thereby accelerating AI enterprises' absorption and internalization and finally boosting their income. Moreover, rapid expansion helps to enhance the flexibility of AI enterprises [12] and improve their ability to respond to complex and changeable markets. Therefore, at the market level, rapid across-industry chain investment can improve the innovation performance of AI enterprises. At the AI enterprise level, the key knowledge, technology, process, structure, and other key resources acquired by the enterprise through investment will be integrated with existing resources. Companies will experience organizational restructuring [13] and finally achieve the ideal organizational structure [14]. In terms of across-industrial-chain investment, it allows enterprises to obtain key resources in the initial stage of expansion. Enterprises try to integrate new resources into their organizations through restructuring. Companies will face "new entrants disadvantage" [15], which undermines corporate innovation performance. The fast cross-industry-chain investment can repeat similar investment actions many times within a predetermined period of time to strengthen the behavior and intention of organizational restructuring [16]. To the greatest extent, it weakens the adverse impact of the "disadvantage of newcomers" caused by organizational restructuring on enterprise innovation performance.

On such basis, the following hypothesis is put forward in this paper:

\section{Research Design}

4.1. Data Acquisition. This paper uses Wind database as the basic data source of empirical research. Wind database includes the financial data of AI enterprises listed on A-share market and is widely used in the research of investment and innovation performance of AI enterprises listed in China [28-30]. At the same time, this paper uses data scraping technology to match the names of AI enterprises listed in Wind database with the information query system of State Administration for Industry and Commerce. This paper searched the names of all wholly owned subsidiaries and tire-one holding subsidiaries of A-share AI enterprises [31]. Then, by matching the main business scope in the industrial and commercial registration of the above-mentioned AI enterprises with the four-digit codes of listed companies, the industry in which these AI enterprises' main business is located is determined.

In addition, this paper draws on the relatively advanced industrial location calculation method proposed by Antràs and Chor [32]. Based on Input-Output Tables of China in 2012 and 2017, the relative position of the industrial chain of 42 industries in the whole national economy is calculated and represented by a numerical value between 0 and 1 . First of all, we can define a basic identity in the Input-Output Table:

$$
Y_{i}=F_{i}+Z_{i}
$$

$Y_{i}$ is the total output of $i$ industry. $F_{i}$ is the part of the $i$ industry output that flows directly to the final consumption and total capital formation. $Z_{i}$ is the part of $i$ industry output consumed by other industries. In a national economic system with $N$ industries, the above equation can be further expounded as

$$
Y_{i}=F_{i}+\underbrace{\sum_{j=1}^{N} d_{i j} F_{j}}_{\text {Direct consumption part in the } i \text { industry output }}+\underbrace{\sum_{j=1}^{N} \sum_{k=1}^{N} d_{i k} d_{k j} F_{j}+\sum_{j=1}^{N} \sum_{k=1}^{N} \sum_{l=1}^{N} d_{i l} d_{l k} d_{k j} F_{j}+\cdots .}_{\text {Indirect consumption part in the } i \text { industry output }}
$$


The calculation method of industrial chain position based on the above formula is as follows (see Table 1 for specific industrial chain position data):

$$
Y=F+D F+D^{2} F+D^{3} F+\cdots=[1-D]^{-1} F .
$$

Based on the industrial chain position formula (3), this paper further calculates the industrial distance between AI enterprises in different industries by means of the difference, still represented by a numerical value between 0 and 1 . Then, using the above-mentioned industry distance from 2014 to 2018, the spanning speed of AI enterprises in across-industrial-chain investment is worked out. In addition, due to the difference in innovation ability training and in R\&D system between the primary, secondary, and tertiary industries, this paper mainly selects the data of A-share listed AI companies in secondary industry for regression analysis and generates a listed AI enterprise database consisting of 275 AI enterprises with data from 2014 to 2018.

\subsection{Variable Structure}

$$
\text { Rdratechain }_{i t}=\sum\left(\operatorname{RDind}_{i k t} * \frac{1}{N_{i t}}\right) .
$$

(1) Dependent variable. According to related research by Bassetti et al. [33], total factor productivity can effectively measure the innovation performance of AI companies. This paper uses the total factor productivity of AI enterprises as a dependent variable of AI enterprise performance.

(2) Explanatory variables. In this paper, the industry score compiled through (3) is used for calculating the absolute value of the difference between the foreign investment industry of listed AI enterprises and its upstream and downstream enterprises, taking it as an across-industry span of investment. Based on the research results of Zhou and Guillen [34], this paper uses the following formula to construct the annual cross-industry chain investment speed variable of listed AI companies:

$$
\operatorname{Speed}_{i t}=\sum\left(\operatorname{IDD}_{i k t} \times \frac{A_{i t}}{T_{i t}}\right) .
$$

$\mathrm{IDD}_{i k t}$ represents the industrial distance of an outward investment behavior. $A_{i t}$ represents the time (year) between this investment behavior and the current year. $T_{i t}$ refers to the time between the first foreign investment of the AI enterprise and the current year.

(3) Moderating variable. The paper firstly takes the number of patents obtained by AI enterprises in that year as the variable of technology accumulation (Lnts). According to the data provided by Wind database, the ratio of R\&D investment to total assets (RDind) of various industries is obtained. After that, this paper weights industries according to how many of them are invested by AI enterprises and then calculates the moderating variable, which is the industrial R\&D investment level (Rdratechain) variable of the industry invested by the AI enterprises, where $\mathrm{N}$ is the total number of cross-industry investments made by AI enterprises. Finally, the paper uses the amount of cross-industry investment made by $\mathrm{AI}$ enterprises as the moderating variable and takes Chainnumber as a variable describing the industrial chain span of cross-industry investment of AI enterprises.

(4) Control variables: Finally, this paper introduces company age (Age), return on assets (Roa), company ownership (Ownership), industry growth rate (Indgrow), total asset logarithmic value (size of the AI enterprise, Size), local GDP (Lngdp), annual dummy variable (Year), and industry dummy variable (Industry) of AI enterprises as controlled variables. All variable names and description are shown in Table 2.

4.3. Measurement Model. According to the above assumptions, databases, and variables, the system generalized method of moments (SYS-GMM) model is used as the main regression model for analysis. The above model uses the lag terms and the difference terms of lag terms as an endogenous tool term, which can adequately solve the endogenous problem of the model [35]. The paper will then group the data based on three layers: $25 \%$ and below, $25 \%-75 \%$, and $75 \%$ and above. The analysis can be shown in the next section for the specific results of checking the regulating effect of related variables. To test the robustness of the model, this paper also uses the panel fixed effect model to analyze the robustness (each model has passed Hausman test).

\section{Analysis of the Regression Results}

5.1. Descriptive Statistics and Correlation Analysis Results. Before verifying the hypothesis, descriptive statistics and correlation analysis were made for each variable in this study, and these results are shown in Tables 3 and 4 .

5.2. Regression Analysis. On the basis of correlation analysis, this study uses SYS-GMM and fixed effect analysis to further verify the research hypotheses.

The SYS-GMM regression results of model (2) in Table 5 show that the across-industrial-chain investment speed of AI enterprises has an impact on innovation performance. There is a significant positive correlation (coefficient $=1.045$, $P<0.01)$ between the across-industrial-chain investment speed of AI enterprises and innovation performance. The results of panel data fixed effect analysis of model (2) in Table 6 also show that there is a significant positive correlation between them (coefficient $=0.959, P<0.01$ ). This shows that, for the purposes of cross-industrial chain investment, the faster the AI enterprises invest, the stronger the innovation performance will be. $\mathrm{H} 1$ is verified. 
TABle 1: Positions in industrial chain.

\begin{tabular}{|c|c|c|c|}
\hline Industry (2012) & Score & Industry (2017) & Score \\
\hline Food and tobacco & 0.363 & Transportation equipment & 0.387 \\
\hline Transportation equipment & 0.313 & Special equipment & 0.375 \\
\hline Processed wood products and furniture & 0.312 & Food and tobacco & 0.343 \\
\hline $\begin{array}{l}\text { Products and services of agriculture, forestry, animal } \\
\text { husbandry, and fishery }\end{array}$ & 0.304 & Processed wood products and furniture & 0.334 \\
\hline Textiles & 0.303 & Gas production and supply & 0.315 \\
\hline Nonmetallic mineral products & 0.294 & Nonmetallic mineral products & 0.313 \\
\hline Electrical machinery and equipment & 0.252 & Water production and supply & 0.309 \\
\hline Special equipment and general equipment & 0.234 & Electrical machinery and equipment & 0.291 \\
\hline Metal products & 0.221 & $\begin{array}{c}\text { Products and services of agriculture, forestry, animal } \\
\text { husbandry, and fishery }\end{array}$ & 0.281 \\
\hline Water production and supply & 0.216 & General equipment & 0.271 \\
\hline Gas production and supply & 0.212 & Textiles & 0.267 \\
\hline Papermaking, printing, and stationery \& sporting goods & 0.209 & Metal products & 0.258 \\
\hline $\begin{array}{l}\text { Mined and processed products from nonmetallic minerals } \\
\text { and other minerals }\end{array}$ & 0.185 & Papermaking, printing, and stationery \& sporting goods & 0.244 \\
\hline $\begin{array}{l}\text { Communication equipment, computers, and other electronic } \\
\text { equipment }\end{array}$ & 0.172 & Smelt and pressed metal products & 0.188 \\
\hline Smelt and pressed metal products & 0.172 & $\begin{array}{c}\text { Communication equipment, computers, and other } \\
\text { electronic equipment }\end{array}$ & 0.183 \\
\hline Chemical products & 0.162 & Chemical products & 0.180 \\
\hline Instrument and apparatus & 0.153 & Oil and gas extraction & 0.165 \\
\hline Oil and gas extraction & 0.150 & Production and supply of electricity and heat & 0.159 \\
\hline Production and supply of electricity and heat & 0.141 & Instrument and apparatus & 0.155 \\
\hline Mined and processed coal products & 0.134 & Mined and processed coal products & 0.131 \\
\hline
\end{tabular}

TABLE 2: Description of specific variables.

\begin{tabular}{lc}
\hline Name of variable & Variable means \\
\hline Ftfp & Total factor productivity \\
Speed & Annual cross-industry chain investment speed of AI enterprise \\
Lnts & AI enterprise technology accumulation \\
Rdratechain & Industrial R\&D investment level of industries invested by AI enterprises \\
Chainnumber & Investment span of industrial chain \\
Age & Establishment time of AI enterprise \\
Roa & ROA (return on asset) \\
Ownership & Enterprise ownership of the AI enterprise \\
Indgrow & Industry growth rate of AI enterprise \\
Size & Scale of AI enterprise \\
Lngdp & GDP of the region where AI enterprise is located \\
Year & Annual dummy variable \\
Industry & Industry dummy variable \\
\hline
\end{tabular}

TABLe 3: Overall descriptive statistics.

\begin{tabular}{|c|c|c|c|c|c|}
\hline Variable name & Observed value & Mean & Standard deviation & Minimum & Maximum \\
\hline Ftfp & 13041 & 0.4605 & 0.4187 & -0.1910 & 2.4390 \\
\hline Speed & 13011 & 0.1649 & 0.1905 & 0.0000 & 2.1331 \\
\hline Lnts & 12994 & 5.6752 & 3.7056 & 0.0000 & 138.000 \\
\hline Rdratechain & 11005 & 0.1131 & 0.0182 & 0.0661 & 0.1811 \\
\hline Chain & 9282 & 2.6787 & 2.8554 & 0.0000 & 12.0370 \\
\hline Age & 13041 & 14.3900 & 5.2485 & 0.0000 & 56.0000 \\
\hline Lnuncer & 13041 & 15.5730 & 1.0448 & 9.7061 & 17.7150 \\
\hline Roa & 13041 & 8.5420 & 20.5960 & -1611.0000 & 1061.6000 \\
\hline Owner & 9911 & 0.3719 & 0.4833 & 0.0000 & 1.0000 \\
\hline Indgrow & 13041 & 12.1500 & 13.9060 & -65.0300 & 70.4600 \\
\hline Lnsize & 12647 & 12.2530 & 1.3751 & 5.5483 & 19.2980 \\
\hline Lngdp & 13041 & 19.4220 & 0.7431 & 15.4400 & 20.4060 \\
\hline
\end{tabular}




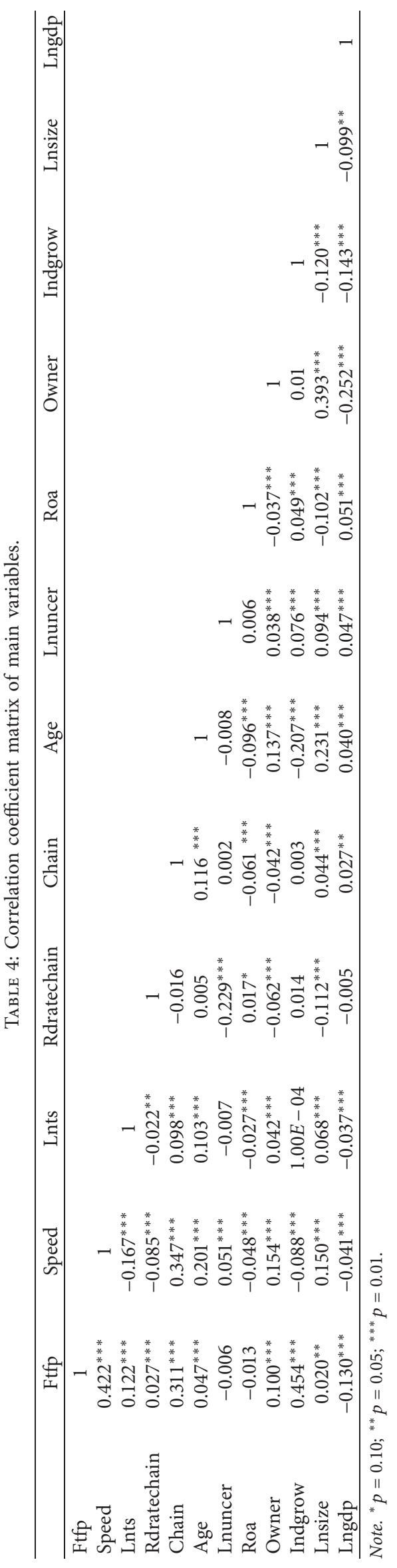


TABLE 5: SYS-GMM analysis results.

\begin{tabular}{|c|c|c|c|}
\hline & $(1)$ & $(2)$ & $(3)$ \\
\hline L.Ftfp & $\begin{array}{c}0.121^{* * *} \\
(0.024)\end{array}$ & $\begin{array}{c}0.059^{* * *} \\
(0.018)\end{array}$ & $\begin{array}{l}0.040^{* *} \\
(0.016)\end{array}$ \\
\hline Lnts & $\begin{array}{c}0.010^{* * *} \\
(0.002)\end{array}$ & $\begin{array}{c}0.022^{* * *} \\
(0.001)\end{array}$ & $\begin{array}{c}0.004^{* * *} \\
(0.001)\end{array}$ \\
\hline Rdratechain & $\begin{array}{c}1.555^{* * *} \\
(0.393) \\
\end{array}$ & $\begin{array}{c}2.323^{* * *} \\
(0.287) \\
\end{array}$ & $\begin{array}{c}1.095^{* * *} \\
(0.304)\end{array}$ \\
\hline Chainnumber & $\begin{array}{c}0.023^{* * *} \\
(0.002)\end{array}$ & $\begin{array}{c}0.010^{* * *} \\
(0.001)\end{array}$ & $\begin{array}{c}0.008^{* * *} \\
(0.001)\end{array}$ \\
\hline Age & $\begin{array}{l}-0.008 \\
(0.014) \\
\end{array}$ & $\begin{array}{c}0.011 \\
(0.010)\end{array}$ & $\begin{array}{c}0.005 \\
(0.009)\end{array}$ \\
\hline Roa & $\begin{array}{l}-2.39 E-04 \\
(2.17 E-04)\end{array}$ & $\begin{array}{l}-1.16 E-04 \\
(1.57 E-04)\end{array}$ & $\begin{array}{l}-1.31 E-04 \\
(1.43 E-04) \\
\end{array}$ \\
\hline Owner & $\begin{array}{c}0.034 \\
(0.045) \\
\end{array}$ & $\begin{array}{c}0.033 \\
(0.033)\end{array}$ & $\begin{array}{c}0.010 \\
(0.030)\end{array}$ \\
\hline Indgrow & $\begin{array}{c}2.12 E-04 \\
(0.001)\end{array}$ & $\begin{array}{c}4.78 E-04 \\
(4.04 E-04)\end{array}$ & $\begin{array}{c}2.91 E-04 \\
(3.68 E-04)\end{array}$ \\
\hline Lnsize & $\begin{array}{c}0.005 \\
(0.014) \\
\end{array}$ & $\begin{array}{c}0.001 \\
(0.010)\end{array}$ & $\begin{array}{c}0.004 \\
(0.009) \\
\end{array}$ \\
\hline Lngdp & $\begin{array}{c}0.193 \\
(0.157) \\
\end{array}$ & $\begin{array}{c}0.154 \\
(0.114) \\
\end{array}$ & $\begin{array}{l}0.172^{*} \\
(0.104)\end{array}$ \\
\hline $\begin{array}{l}\text { Year } \\
\text { Industry } \\
\text { Speed }\end{array}$ & $\begin{array}{l}\text { Control } \\
\text { Control }\end{array}$ & $\begin{array}{c}\text { Control } \\
\text { Control } \\
1.045^{* * *} \\
(0.023) \\
\end{array}$ & $\begin{array}{c}\text { Control } \\
\text { Control } \\
-0.213^{*} \\
(0.122) \\
\end{array}$ \\
\hline Speed ${ }^{*}$ Lnts & & & $\begin{array}{c}0.129^{* * *} \\
(0.005)\end{array}$ \\
\hline Speed* Rdratechain & & & $\begin{array}{c}4.579^{* * *} \\
(1.025) \\
\end{array}$ \\
\hline Obs\# & 6350 & 6350 & 6350 \\
\hline Firms & 1586 & 1586 & 1586 \\
\hline Wald chi $^{2}$ & 336.1 & 2706.56 & 3965.19 \\
\hline
\end{tabular}

Note. ${ }^{*} p=0.10 ;{ }^{* *} p=0.05 ;{ }^{* * *} p=0.01$.

The SYS-GMM regression results of model (3) in Table 5 show that the technology accumulation of the AI enterprises has an impact on the relationship between the across-industrial-chain investment speed and innovation performance. Technology accumulation of AI enterprises can significantly and positively regulate the relationship between the across-industrial-chain investment speed and innovation performance (coefficient $=0.129, P<0.01$ ). The results of panel data fixed effect analysis of model (3) in Table 6 also show that the adjustment relationship exists significantly (coefficient $=0.109, P<0.01$ ). After analyzing the data separately by SYS-GMM (as shown in Table 7), it can be found that as the technology accumulation of AI enterprises increases, the regression coefficients also gradually increase (the coefficients of $25 \%$ quantile and below $=0.433$, the coefficients of $25 \%-75 \%$ quantile $=1.244$, and the coefficients of $75 \%$ quantile and above $=1.392 ; P<0.01)$. The fixed effect grouping analysis results of panel data in Table 8 are also significant (coefficients of $25 \%$ quantile and below $=0.310$, coefficients of $25 \%-75 \%$ quantile $=1.469$, and coefficients of $75 \%$ quantile and above $=1.577 ; P<0.01)$. The above results show that in the rapid cross-industrial-chain
TABLE 6: Fixed effect analysis results of panel data.

\begin{tabular}{|c|c|c|c|}
\hline & $(1)$ & $(2)$ & (3) \\
\hline Lnts & $\begin{array}{c}0.011^{* * *} \\
(0.001)\end{array}$ & $\begin{array}{c}0.020^{* * * *} \\
(0.001)\end{array}$ & $\begin{array}{c}0.006^{* * *} \\
(0.001)\end{array}$ \\
\hline Rdratechain & $\begin{array}{c}1.119^{* * *} \\
(0.302)\end{array}$ & $\begin{array}{c}2.022^{* * * *} \\
(0.228)\end{array}$ & $\begin{array}{c}0.959^{* * *} \\
(0.245)\end{array}$ \\
\hline Chainnumber & $\begin{array}{c}0.022^{* * *} \\
(0.001) \\
\end{array}$ & $\begin{array}{c}0.009^{* * *} \\
(0.001)\end{array}$ & $\begin{array}{c}0.008^{* * *} \\
(0.001) \\
\end{array}$ \\
\hline Age & $\begin{array}{l}-0.006 \\
(0.011) \\
\end{array}$ & $\begin{array}{l}-0.009 \\
(0.008) \\
\end{array}$ & $\begin{array}{l}-0.006 \\
(0.007)\end{array}$ \\
\hline Roa & $\begin{array}{c}-2.88-E-05 \\
(1.92 E-04)\end{array}$ & $\begin{array}{c}2.57 E-05 \\
(1.44 E-04)\end{array}$ & $\begin{array}{c}-2.65-E-05 \\
(1.34 E-04)\end{array}$ \\
\hline Owner & $\begin{array}{l}-0.030 \\
(0.030) \\
\end{array}$ & $\begin{array}{c}-0.018 \\
(0.023) \\
\end{array}$ & $\begin{array}{l}-0.018 \\
(0.021)\end{array}$ \\
\hline Indgrow & $\begin{array}{c}6.62 E-05 \\
(4.05 E-04)\end{array}$ & $\begin{array}{c}4.17 E-04 \\
(3.04 E-04) \\
\end{array}$ & $\begin{array}{c}0.001^{* *} \\
(2.82 E-04)\end{array}$ \\
\hline Lnsize & $\begin{array}{c}-0.017^{*} \\
(0.009)\end{array}$ & $\begin{array}{c}-0.015^{* *} \\
(0.007) \\
\end{array}$ & $\begin{array}{c}-0.012^{*} \\
(0.006) \\
\end{array}$ \\
\hline Lngdp & $\begin{array}{c}0.117 \\
(0.110) \\
\end{array}$ & $\begin{array}{c}0.134 \\
(0.082) \\
\end{array}$ & $\begin{array}{c}0.111 \\
(0.076) \\
\end{array}$ \\
\hline Year & Control & Control & Control \\
\hline Industry & Control & Control & Control \\
\hline Speed & & $\begin{array}{c}0.959^{* * *} \\
(0.018)\end{array}$ & $\begin{array}{l}-0.125 \\
(0.098)\end{array}$ \\
\hline Speed ${ }^{*}$ Lnts & & & $\begin{array}{c}0.109^{* * *} \\
(0.004)\end{array}$ \\
\hline Speed*Rdratechain & & & $\begin{array}{c}3.974^{* * *} \\
(0.818)\end{array}$ \\
\hline Obs\# & 6363 & 6352 & 6352 \\
\hline Firms & 1586 & 1586 & 1586 \\
\hline$R^{2}$ & 0.074 & 0.414 & 0.496 \\
\hline$F$ & 31.87 & 258.57 & 311.29 \\
\hline
\end{tabular}

Note. ${ }^{*} p=0.10 ;{ }^{* *} p=0.05 ;{ }^{* * *} p=0.01$.

investment, the AI enterprises with rich technology accumulation can benefit from it and therefore improve innovation performance, and $\mathrm{H} 2$ is verified.

The SYS-GMM regression results of model (3) in Table 5 show that the industrial R\&D investment level of industries invested by the AI enterprises can significantly and positively adjust the relationship between the across-industrial-chain investment speed and innovation performance (coefficient $=4.579 ; P<0.01)$. The results of panel data fixed effect analysis of model (6) also show that the adjustment relationship exists significantly (coefficient $=3.974 ; P<0.01$ ). After analyzing the data separately by SYS-GMM (as shown in Table 9), it can be found that as the industrial R\&D investment level of industries invested by the AI enterprises increases, the regression coefficients also gradually increase (the coefficients of $25 \%$ quantile and below $=0.971$, the coefficients of $25 \%-75 \%$ quantile $=1.061$, the coefficients of $75 \%$ quantile and above $=1.072 ; P<0.01)$. The fixed effect grouping analysis results of panel data in Table 10 partly verify the robustness of the above results (the coefficients of $25 \%$ quantile and below $=0.837$, the coefficients of $25 \%-75 \%$ quantile $=1.020$, and the coefficients of $75 \%$ quantile and above $=1.000 ; P<0.01)$. The above results show that for the purposes of the rapid cross-industrial-chain investment, the 
TABLE 7: SYS-GMM Analysis results (hypothesis 2).

\begin{tabular}{|c|c|c|c|}
\hline & $\begin{array}{l}\text { (1) } \\
\text { Lnts of less than or equal to } 25 \%\end{array}$ & $\begin{array}{c}(2) \\
\text { Lnts of } 25 \%-75 \%\end{array}$ & $\begin{array}{c}\text { (3) } \\
\text { Lnts of larger than or equal to } 75 \%\end{array}$ \\
\hline L.Ftfp & $\begin{array}{c}0.187^{* * *} \\
(0.047) \\
\end{array}$ & $\begin{array}{l}0.043^{*} \\
(0.025) \\
\end{array}$ & $\begin{array}{c}0.071^{*} \\
(0.037) \\
\end{array}$ \\
\hline Lnts & $\begin{array}{c}0.021^{* * *} \\
(0.003)\end{array}$ & $\begin{array}{c}0.018^{* * *} \\
(0.002) \\
\end{array}$ & $\begin{array}{c}0.024^{* * *} \\
(0.002)\end{array}$ \\
\hline Rdratechain & $\begin{array}{c}0.513 \\
(0.726)\end{array}$ & $\begin{array}{c}2.336^{* * *} \\
(0.353)\end{array}$ & $\begin{array}{c}2.517^{* * *} \\
(0.588)\end{array}$ \\
\hline Chainnumber & $\begin{array}{c}0.013^{* * *} \\
(0.003)\end{array}$ & $\begin{array}{c}0.005^{* * *} \\
(0.002)\end{array}$ & $\begin{array}{c}0.012^{* * *} \\
(0.002)\end{array}$ \\
\hline Age & $\begin{array}{l}-0.269 \\
(0.477) \\
\end{array}$ & $\begin{array}{l}-0.185 \\
(0.208)\end{array}$ & $\begin{array}{l}-0.042 \\
(0.034)\end{array}$ \\
\hline Roa & $\begin{array}{l}-0.001 \\
(0.002)\end{array}$ & $\begin{array}{c}0.001^{* *} \\
(4.93 E-04)\end{array}$ & $\begin{array}{l}-2.00 E-04 \\
(1.63 E-04)\end{array}$ \\
\hline Owner & $\begin{array}{c}0.036 \\
(0.090)\end{array}$ & $\begin{array}{c}0.029 \\
(0.043) \\
\end{array}$ & $\begin{array}{c}0.034 \\
(0.054) \\
\end{array}$ \\
\hline Indgrow & $\begin{array}{c}0.001 \\
(0.001) \\
\end{array}$ & $\begin{array}{c}2.62 E-04 \\
(0.001)\end{array}$ & $\begin{array}{c}2.66 E-04 \\
(0.001)\end{array}$ \\
\hline Lnsize & $\begin{array}{l}-0.017 \\
(0.033)\end{array}$ & $\begin{array}{c}0.005 \\
(0.013) \\
\end{array}$ & $\begin{array}{l}-0.004 \\
(0.017)\end{array}$ \\
\hline Lngdp & $\begin{array}{c}0.182 \\
(0.319)\end{array}$ & $\begin{array}{c}0.146 \\
(0.170)\end{array}$ & $\begin{array}{c}0.205 \\
(0.199)\end{array}$ \\
\hline $\begin{array}{l}\text { Year } \\
\text { Industry } \\
\text { Speed }\end{array}$ & $\begin{array}{c}\text { Control } \\
\text { Control } \\
0.433^{* * *} \\
(0.049)\end{array}$ & $\begin{array}{c}\text { Control } \\
\text { Control } \\
1.244^{* * *} \\
(0.031)\end{array}$ & $\begin{array}{c}\text { Control } \\
\text { Control } \\
1.392^{* * *} \\
(0.046)\end{array}$ \\
\hline $\begin{array}{l}\text { Obs\# } \\
\text { Firms } \\
\text { Wald chi2 }\end{array}$ & $\begin{array}{c}1192 \\
504 \\
4179.63\end{array}$ & $\begin{array}{c}3389 \\
1190 \\
21220.3\end{array}$ & $\begin{array}{c}1769 \\
760 \\
1258.27\end{array}$ \\
\hline
\end{tabular}

Note. ${ }^{*} p=0.10 ;{ }^{* *} p=0.05 ;{ }^{* * *} p=0.01$.

TABLE 8: Fixed effect analysis results of panel data (hypothesis 2).

\begin{tabular}{|c|c|c|c|}
\hline & $\begin{array}{l}\text { (1) } \\
\text { Lnts of less than or equal to } 25 \%\end{array}$ & $\begin{array}{c}(2) \\
\text { Lnts of } 25 \%-75 \%\end{array}$ & $\begin{array}{c}\text { (3) } \\
\text { Lnts of larger than or equal to } 75 \%\end{array}$ \\
\hline Lnts & $\begin{array}{c}0.017 \\
(0.029)\end{array}$ & $\begin{array}{l}0.013^{* *} \\
(0.005)\end{array}$ & $\begin{array}{l}0.008^{*} \\
(0.005)\end{array}$ \\
\hline Rdratechain & $\begin{array}{c}0.043 \\
(0.615)\end{array}$ & $\begin{array}{c}2.416^{* * *} \\
(0.286)\end{array}$ & $\begin{array}{c}2.577^{* * *} \\
(0.525)\end{array}$ \\
\hline Chainnumber & $\begin{array}{c}0.014^{* * *} \\
(0.003)\end{array}$ & $\begin{array}{c}4.50 E-04 \\
(0.001)\end{array}$ & $\begin{array}{c}0.011^{* * *} \\
(0.002)\end{array}$ \\
\hline Age & $\begin{array}{c}-0.018 \\
(0.019)\end{array}$ & $\begin{array}{l}-0.014 \\
(0.010)\end{array}$ & $\begin{array}{c}-0.037^{* * *} \\
(0.014)\end{array}$ \\
\hline Roa & $\begin{array}{l}-0.001 \\
(0.001)\end{array}$ & $\begin{array}{c}0.002^{* * *} \\
(4.14 E-04)\end{array}$ & $\begin{array}{c}-1.63-E-04 \\
(1.51 E-04)\end{array}$ \\
\hline Owner & $\begin{array}{l}-0.045 \\
(0.068) \\
\end{array}$ & $\begin{array}{c}-0.018 \\
(0.030)\end{array}$ & $\begin{array}{c}0.042 \\
(0.041) \\
\end{array}$ \\
\hline Indgrow & $\begin{array}{l}-0.001 \\
(0.001)\end{array}$ & $\begin{array}{c}4.86 E-04 \\
(3.79 E-04)\end{array}$ & $\begin{array}{c}-1.79 E-04 \\
(0.001)\end{array}$ \\
\hline Lnsize & $\begin{array}{c}-0.049^{* *} \\
(0.024)\end{array}$ & $\begin{array}{c}-0.011 \\
(0.008)\end{array}$ & $\begin{array}{l}-0.021 \\
(0.016)\end{array}$ \\
\hline Lngdp & $\begin{array}{c}0.236 \\
(0.218) \\
\end{array}$ & $\begin{array}{l}0.207^{*} \\
(0.118) \\
\end{array}$ & $\begin{array}{c}0.393^{* *} \\
(0.162)\end{array}$ \\
\hline $\begin{array}{l}\text { Year } \\
\text { Industry }\end{array}$ & $\begin{array}{l}\text { Control } \\
\text { Control }\end{array}$ & $\begin{array}{l}\text { Control } \\
\text { Control }\end{array}$ & $\begin{array}{l}\text { Control } \\
\text { Control }\end{array}$ \\
\hline
\end{tabular}


Table 8: Continued.

\begin{tabular}{lccc}
\hline & $(1)$ & $(2)$ & $(3)$ \\
& Lnts of less than or equal to $25 \%$ & Lnts of $25 \%-75 \%$ & Lnts of larger than or equal to $75 \%$ \\
\hline \multirow{2}{*}{ Speed } & $0.310^{* * *}$ & $1.469^{* * *}$ & $1.577^{* * *}$ \\
& $(0.055)$ & $(0.029)$ & $(0.048)$ \\
\hline Obs\# & 1192 & 3391 & 1769 \\
$R^{2}$ & 0.101 & 0.567 & 0.586 \\
Firms & 504 & 1190 & 760 \\
$F$ & 5.83 & 220.79 & 108.46 \\
\hline
\end{tabular}

Note. ${ }^{*} p=0.10 ;{ }^{* *} p=0.05 ;{ }^{* * *} p=0.01$.

TABLE 9: SYS-GMM analysis results (hypothesis 3).

(1) (2) (3)

Rdratechain of less than or equal to $25 \% \quad$ Rdratechain of $25 \%-75 \% \quad$ Rdratechain of larger than or equal to $75 \%$

\begin{tabular}{|c|c|c|c|}
\hline L.Ftfp & $\begin{array}{c}0.155^{* * *} \\
(0.048)\end{array}$ & $\begin{array}{c}0.033 \\
(0.023)\end{array}$ & $\begin{array}{c}0.013 \\
(0.039)\end{array}$ \\
\hline Lnts & $\begin{array}{c}0.022^{* * *} \\
(0.003)\end{array}$ & $\begin{array}{c}0.021^{* * *} \\
(0.002)\end{array}$ & $\begin{array}{c}0.023^{* * *} \\
(0.002)\end{array}$ \\
\hline Rdratechain & $\begin{array}{c}7.131^{* * *} \\
(2.200)\end{array}$ & $\begin{array}{c}2.378^{* * *} \\
(0.366)\end{array}$ & $\begin{array}{c}28.033 \\
(39.995)\end{array}$ \\
\hline Chainnumber & $\begin{array}{c}0.014^{* * *} \\
(0.003)\end{array}$ & $\begin{array}{c}0.011^{* * *} \\
(0.002)\end{array}$ & $\begin{array}{c}0.008^{* * *} \\
(0.003)\end{array}$ \\
\hline Age & $\begin{array}{c}-0.081^{* * *} \\
(0.031) \\
\end{array}$ & $\begin{array}{l}-0.082 \\
(0.218)\end{array}$ & $\begin{array}{l}-0.048 \\
(0.062)\end{array}$ \\
\hline Roa & $\begin{array}{c}-2.40-E-04 \\
(1.81 E-04)\end{array}$ & $\begin{array}{c}4.65 E-04 \\
(0.001)\end{array}$ & $\begin{array}{l}0.003^{* *} \\
(0.001)\end{array}$ \\
\hline Owner & $\begin{array}{c}0.156^{*} \\
(0.086) \\
\end{array}$ & $\begin{array}{l}-0.026 \\
(0.041) \\
\end{array}$ & $\begin{array}{l}0.127^{*} \\
(0.066)\end{array}$ \\
\hline Indgrow & $\begin{array}{l}-0.001 \\
(0.001) \\
\end{array}$ & $\begin{array}{c}0.001 \\
(0.001) \\
\end{array}$ & $\begin{array}{l}-0.002 \\
(0.005)\end{array}$ \\
\hline Lnsize & $\begin{array}{l}-0.001 \\
(0.021) \\
\end{array}$ & $\begin{array}{c}0.003 \\
(0.014) \\
\end{array}$ & $\begin{array}{l}-0.019 \\
(0.023) \\
\end{array}$ \\
\hline Lngdp & $\begin{array}{l}0.620^{* *} \\
(0.282)\end{array}$ & $\begin{array}{c}0.054 \\
(0.154) \\
\end{array}$ & $\begin{array}{l}-0.088 \\
(0.255)\end{array}$ \\
\hline $\begin{array}{l}\text { Year } \\
\text { Industry } \\
\text { Speed }\end{array}$ & $\begin{array}{c}\text { Control } \\
\text { Control } \\
0.971^{* * *} \\
(0.055) \\
\end{array}$ & $\begin{array}{c}\text { Control } \\
\text { Control } \\
1.061^{* * *} \\
(0.029) \\
\end{array}$ & $\begin{array}{c}\text { Control } \\
\text { Control } \\
1.072^{* * *} \\
(0.048) \\
\end{array}$ \\
\hline $\begin{array}{l}\text { Obs\# } \\
\text { Firms } \\
\text { Wald chi }\end{array}$ & $\begin{array}{c}1589 \\
657 \\
478.31\end{array}$ & $\begin{array}{c}3385 \\
1170 \\
21289.59\end{array}$ & $\begin{array}{c}1376 \\
567 \\
7808.14\end{array}$ \\
\hline
\end{tabular}

Note. ${ }^{*} p=0.10 ;{ }^{* *} p=0.05 ;{ }^{* * *} p=0.01$.

TABLe 10: Fixed effect analysis results of panel data (hypothesis 3).
(1)
(2)
(3)

Rdratechain of less than or equal to $25 \% \quad$ Rdratechain of $25 \%-75 \% \quad$ Rdratechain of larger than or equal to $75 \%$

\begin{tabular}{lccc}
\hline \multirow{2}{*}{ Lnts } & $0.018^{* * *}$ & $0.022^{* * *}$ & $0.020^{* * *}$ \\
& $(0.002)$ & $(0.001)$ & $(0.002)$ \\
\hline \multirow{2}{*}{ Rdratechain } & -3.133 & 1.191 & -10.128 \\
& $(3.843)$ & $(1.518)$ & $(174.068)$ \\
\hline \multirow{2}{*}{ Chainnumber } & $0.012^{* * *}$ & $0.010^{* * *}$ & $(0.001)$ \\
\hline \multirow{2}{*}{ Age } & $(0.003)$ & $-0.023^{* *}$ & $\left(0.008^{* * *}\right.$ \\
& 0.009 & $(0.011)$ & -0.033 \\
\hline
\end{tabular}


TABle 10: Continued.

(1) (2) (3)

Rdratechain of less than or equal to $25 \% \quad$ Rdratechain of $25 \%-75 \% \quad$ Rdratechain of larger than or equal to $75 \%$

\begin{tabular}{lccc}
\hline \multirow{2}{*}{ Roa } & $-9.37 E-05$ & 0.001 & $0.002^{* *}$ \\
& $(1.59 E-04)$ & $(0.001)$ & $(0.001)$ \\
\hline \multirow{2}{*}{ Owner } & -0.012 & -0.015 & -0.009 \\
& $(0.059)$ & $(0.032)$ & $(0.052)$ \\
\hline \multirow{2}{*}{ Indgrow } & $-1.22-E-04$ & 0.001 & $-0.002^{* *}$ \\
& $(0.001)$ & $(4.29 E-04)$ & $(0.001)$ \\
\hline \multirow{2}{*}{ Lnsize } & $-0.026^{*}$ & -0.015 & -0.028 \\
& $(0.014)$ & $(0.011)$ & $(0.018)$ \\
Lngdp & 0.033 & $0.265^{* *}$ & -0.135 \\
\hline Year & $(0.214)$ & $(0.130)$ & $(0.164)$ \\
Industry & Control & Control & Control \\
Speed & Control & Control & Control \\
Obs\# & $0.837^{* * *}$ & $1.020^{* * *}$ & $1.000^{* * *}$ \\
$R^{2}$ & $(0.042)$ & $(0.026)$ & $(0.046)$ \\
Firms & 1590 & 3385 & 1377 \\
$F$ & 0.365 & 0.463 & 0.423 \\
\hline
\end{tabular}

Note. ${ }^{*} p=0.10 ;{ }^{* *} p=0.05 ;{ }^{* * *} p=0.01$

TABLE 11: SYS-GMM analysis results (hypothesis 4).
(1)
(2)
(3)

Chainnumber of less than or equal to $25 \%$ Chainnumber of $25 \%-75 \%$ Chainnumber of larger than or equal to $75 \%$

\begin{tabular}{|c|c|c|c|}
\hline L.Ftfp & $\begin{array}{c}\left(0.174^{* * *}\right) \\
-0.024\end{array}$ & $\begin{array}{l}(0.015) \\
-0.024\end{array}$ & $\begin{array}{c}(0.013) \\
-0.095\end{array}$ \\
\hline Lnts & $\begin{array}{c}\left.0.010^{* * *}\right) \\
-0.001\end{array}$ & $\begin{array}{c}\left(0.028^{* * *}\right) \\
-0.002 \\
\end{array}$ & $\begin{array}{c}\left(0.020^{* * *}\right) \\
-0.005\end{array}$ \\
\hline Rdratechain & $\begin{array}{c}\left(1.937^{* * *}\right) \\
-0.341\end{array}$ & $\begin{array}{c}\left(2.542^{* * *}\right) \\
-0.438\end{array}$ & $\begin{array}{l}(0.744) \\
-1.210\end{array}$ \\
\hline Chainnumber & $\begin{array}{c}\left.0.013^{* * *}\right) \\
-0.001 \\
\end{array}$ & $\begin{array}{c}\left(0.011^{* * *}\right) \\
-0.002\end{array}$ & $\begin{array}{l}(0.010) \\
-0.004 \\
\end{array}$ \\
\hline Age & $\begin{array}{c}(0.016) \\
-0.041 \\
\end{array}$ & $\begin{array}{r}(0.024) \\
-0.027 \\
\end{array}$ & $\begin{array}{r}(0.062) \\
-0.053 \\
\end{array}$ \\
\hline Roa & $\begin{array}{c}\left(0.002^{* * *}\right) \\
-0.001 \\
\end{array}$ & $\begin{array}{c}(1.68 E-04) \\
1.77 E-04\end{array}$ & $\begin{array}{c}(4.39 E-04) \\
-0.002\end{array}$ \\
\hline Owner & $\begin{array}{r}(0.010) \\
-0.040 \\
\end{array}$ & $\begin{array}{c}(0.025) \\
-0.049 \\
\end{array}$ & $\begin{array}{c}(0.002) \\
-0.133 \\
\end{array}$ \\
\hline Indgrow & $\begin{array}{c}(0.001) \\
4.89 E-04\end{array}$ & $\begin{array}{c}(2.34 E-04) \\
-0.001\end{array}$ & $\begin{array}{l}(0.001) \\
-0.002 \\
\end{array}$ \\
\hline Lnsize & $\begin{array}{r}(0.001) \\
-0.014 \\
\end{array}$ & $\begin{array}{r}(0.003) \\
-0.014 \\
\end{array}$ & $\begin{array}{r}(0.048) \\
-0.055 \\
\end{array}$ \\
\hline Lngdp & $\begin{array}{c}(0.070) \\
-0.151\end{array}$ & $\begin{array}{c}(0.192) \\
-0.182\end{array}$ & $\begin{array}{l}(0.261) \\
-0.604\end{array}$ \\
\hline $\begin{array}{l}\text { Year } \\
\text { Industry } \\
\text { Speed }\end{array}$ & $\begin{array}{c}\text { Control } \\
\text { Control } \\
\left(0.552^{* * *}\right) \\
-0.033 \\
\end{array}$ & $\begin{array}{c}\text { Control } \\
\text { Control } \\
\left(1.274^{* * *}\right) \\
-0.032 \\
\end{array}$ & $\begin{array}{c}\text { Control } \\
\text { Control } \\
\left(0.772^{* * *}\right) \\
-0.068 \\
\end{array}$ \\
\hline $\begin{array}{l}\text { Obs\# } \\
\text { Firms } \\
\text { Wald } \mathrm{chi}^{2}\end{array}$ & $\begin{array}{c}2840 \\
1210 \\
695.68\end{array}$ & $\begin{array}{c}3166 \\
1003 \\
2097.99\end{array}$ & $\begin{array}{c}344 \\
145 \\
200.77\end{array}$ \\
\hline
\end{tabular}

Note. ${ }^{*} p=0.10 ;{ }^{* *} p=0.05 ;{ }^{* * *} p=0.01$. 
TABLE 12: Fixed effect analysis results of panel data (hypothesis 4).

(1)

Chainnumber of less than or equal to $25 \%$ Chainnumber

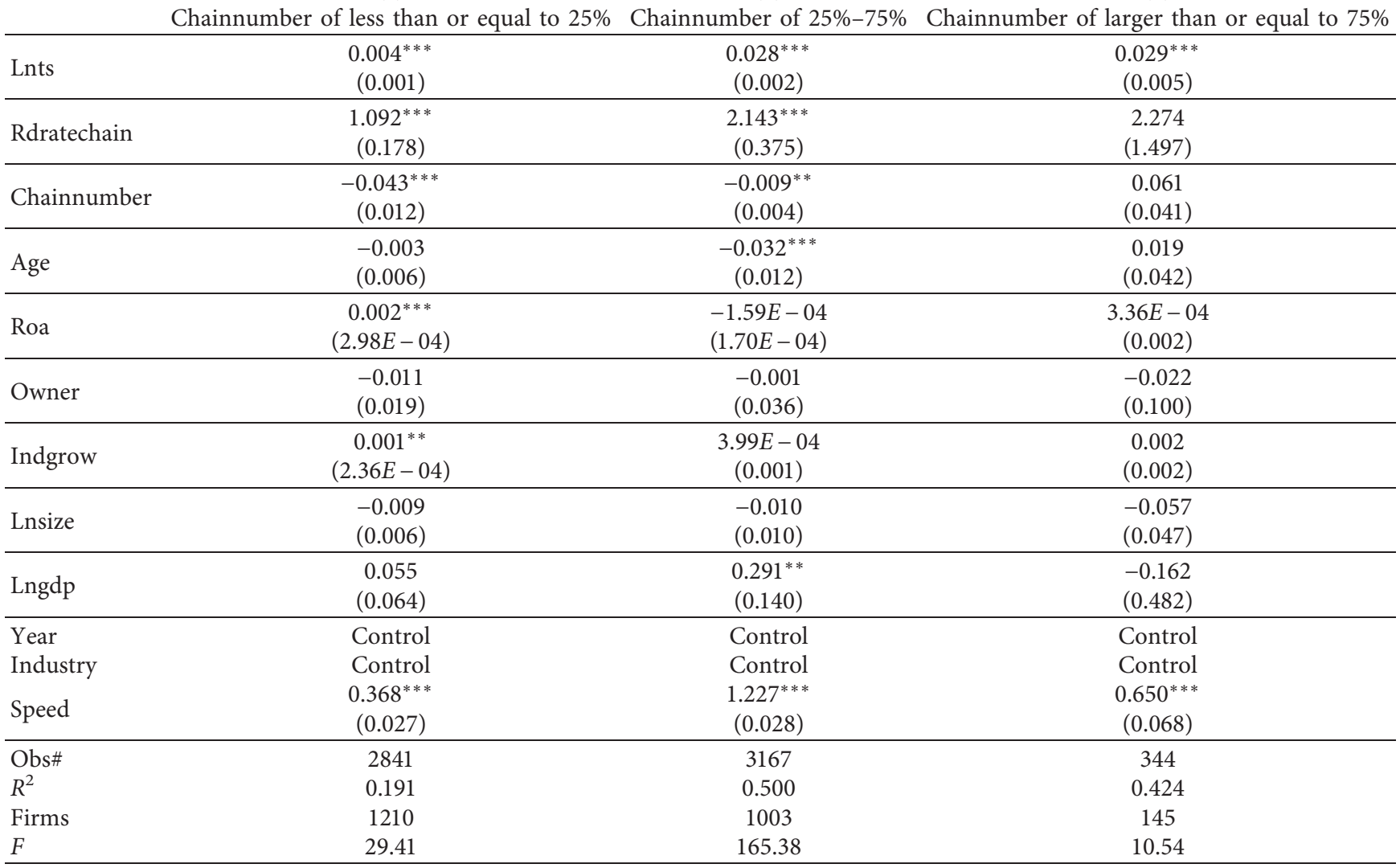

Note. ${ }^{*} p=0.10 ;{ }^{* *} p=0.05 ;{ }^{* * *} p=0.01$

AI enterprises with higher industrial R\&D investment levels in their invested industries can get more benefits and improve their innovation performance, and therefore $\mathrm{H} 3$ is verified.

The influence of industrial chain investment span on the relationship between across-industrial-chain investment speed and innovation performance of $\mathrm{AI}$ enterprises is shown as follows: the industrial chain investment span is subjected to data grouping based on three layers: $25 \%$ and below, 25\%-75\%, and 75\% and above. After analyzing the data separately by SYS-GMM (as shown in Table 11), it can be found that as the industrial chain investment span expands, the regression coefficients show the trend of first increasing and then decreasing (the coefficients of $25 \%$ quantile and below $=0.552$, the coefficients of $25 \%-75 \%$ quantile $=1.274$, and the coefficients of $75 \%$ quantile and above $=0.772 ; P<0.01)$. The fixed effect grouping analysis results of panel data in Table 12 are also significant (coefficients of $25 \%$ quantile and below $=0.368$, coefficients of $25 \%-75 \%$ quantile $=1.227$, and coefficients of $75 \%$ quantile and above $=0.650 ; P<0.01)$. The above results show that in the rapid cross-industrial-chain investment, the more moderate the industrial chain span of the AI enterprises is, the better their innovation performance can be improved, and therefore $\mathrm{H} 4$ is verified.

\section{Conclusion}

Based on the organizational learning theory, this paper discusses the influence of cross-industrial-chain investment speed on the innovation performance of the AI enterprises from the perspective of time dimension of AI enterprise strategy, and it uses the investment data of Chinese listed AI enterprises and the financial data of AI enterprises for empirical test. Four conclusions are drawn as follows: (1) The innovation performance of AI enterprises can be improved by increasing the cross-industrial chain investment speed. (2) Compared with AI enterprises with poor technology accumulation, those with rich technology accumulation can improve their innovation performance with rapid cross-industrial-chain investment. (3) The higher the R\&D investment levels of target industries invested by the AI enterprises in an acrossindustrial-chain mode, the faster the innovation performance of the AI enterprises can be improved by increasing the industrial chain investment speed. (4) Compared with shorter or longer industrial chain investment span, under the condition of moderate industrial chain investment span, the innovation performance of AI enterprises can be further improved by increasing the industrial chain investment spanning speed. 
To a great extent, the selection of investment mode can determine whether the AI enterprises can seize the first opportunity in a market filled with fierce competition. The fast cross-industrial chain investment model can ensure the realization of investment effect and the improvement of innovation performance of AI enterprise, from three aspects: market, AI enterprises, and managers. "Fast" here specifically refers to three aspects: The first is being fast in seizing the opportunity, i.e., to make decisive action and rapid deployment when faced with opportunities so as to seize the initiative before competitors and other potential competitive market players are aware. The second is being fast in investment speed; i.e., after making the decision of cross-industrial-chain investment, the focus AI enterprises and target AI enterprises should actively negotiate and communicate and promote investment transactions in a short time. The third is being fast in the integration process after investment. Since the integration effect after investment determines the final investment performance of the AI enterprises, whether the key resources of target AI enterprises can be fully understood, absorbed, and internalized becomes the biggest challenge. Therefore, the AI enterprises must accelerate the integration process to enhance their innovation performance.

After determining the rapid investment mode, the AI enterprises need to select across-industrial-chain investment targets and take the R\&D investment intensity of the target industries as an important consideration index. The target AI enterprises with strong learning ability can learn the organizational behavior characteristics of the focus AI enterprises after contacting the latter to ensure smooth development of organizational integration after investment. Furthermore, the focus AI enterprises are more likely to benefit from government subsidies, patent protection, tax reduction, and innovation in target industries. At this time, it is also necessary to take the industrial span as one of the important target selection criteria. The AI enterprises should choose a moderate industrial chain investment span, since the expected improvement of innovation performance will not be produced by rapid cross-industrial chain investment if the span is too long or too short. In regard to the selection of a suitable industrial chain, the AI enterprises should adhere to a selection principle of "put quality before quantity," to select the best one and conduct in-depth research on target industries to comprehensively and deeply understand the market status, development prospects, and potential investment returns of target industries. In addition, the AI enterprises should also dare to invest and be good at investing to avoid the short investment span of industrial chains, and understand the industries with development potential and market prospects to the greatest extent through information collection, industry research, and expert consultation.

After selecting suitable investment targets, the AI enterprises need to pay attention to the impact of their own innovation ability on rapid cross-industrial chain investment. The AI enterprises with rich technology accumulation should give full play to their technological advantages; fully understand, absorb, and internalize the key resources of the target AI enterprises during rapid cross-industrial-chain investment with strong learning ability; and quickly integrate these resources into organization routines to form organizational structures and process that serve to enhance their own innovation ability. Those with poor technology accumulation may also need to focus on whether their learning ability can match the target AI enterprises. All AI enterprises should learn from existing partners and even competitors. In addition, after determining the investment target, the AI enterprises can build a resource management system matching the key resources of the target AI enterprises in advance. In this way, before the investment is completed, they can fully prepare for the coming organizational integration and reconstruction, which can help make up for the disadvantage brought by weak technology accumulation.

\section{Data Availability}

The data used to support the findings of this study are included within the article.

\section{Conflicts of Interest}

There are no potential conflicts of interest with regard to the content of the manuscript.

\section{Acknowledgments}

Throughout the study period, the authors and their institutions received funding from government agencies. This work was supported by the Chinese Project of National Natural Science Fund under Grant [71573023].

\section{References}

[1] P. Tambe, P. Cappelli, and V. Yakubovich, "Artificial intelligence in human resources management: challenges and a path forward," California Management Review, vol. 61, no. 4, pp. 15-42, 2019.

[2] I. Ahmed, A. Ahmad, F. Piccialli, A. K. Sangaiah, and G. Jeon, "A robust features-based person tracker for overhead views in industrial environment," IEEE Internet of Things Journal, vol. 5, no. 3, pp. 1598-1605, 2017.

[3] O. Zawacki-Richter, V. I. Marín, M. Bond, and F. Gouverneur, "Systematic review of research on artificial intelligence applications in higher education-where are the educators?" International Journal of Educational Technology in Higher Education, vol. 16, no. 1, pp. 1-27, 2019.

[4] D. M. Dimiduk, E. A. Holm, and S. R. Niezgoda, "Perspectives on the impact of machine learning, deep learning, and artificial intelligence on materials, processes, and structures engineering," Integrating Materials and Manufacturing Innovation, vol. 7, no. 3, pp. 157-172, 2018.

[5] N. Hashai, M. Kafouros, and P. J. Buckley, "The performance implications of speed, regularity, and duration in alliance portfolio expansion," Journal of Management, vol. 44, no. 2, pp. 707-731, 2018.

[6] S. Kunisch, J. M. Bartunek, J. Mueller, and Q. N. Huy, "Time in strategic change research," The Academy of Management Annals, vol. 11, no. 2, pp. 1005-1064, 2017. 
[7] D. Wang, D. Guo, and H. Zhang, "Spatial temporal data visualization in emergency management: a view from datadriven decision," 2017.

[8] S. Raisch and S. Krakowski, "Artificial intelligence and management: the automation-augmentation paradox," Academy of Management Review, vol. 46, no. 1, pp. 192-210, 2021.

[9] Y. Duan, J. S. Edwards, and Y. K. Dwivedi, "Artificial intelligence for decision making in the era of Big Data-evolution, challenges and research agenda," International Journal of Information Management, vol. 48, pp. 63-71, 2019.

[10] P. Neirotti and D. Pesce, "ICT-based innovation and its competitive outcome: the role of information intensity," European Journal of Innovation Management, vol. 22, no. 2, pp. 383-404, 2019.

[11] W. Shi, J. Sun, and J. E. Prescott, "A temporal perspective of merger and acquisition and strategic alliance initiatives," Journal of Management, vol. 38, no. 1, pp. 164-209, 2012.

[12] D. J. Teece, "Explicating dynamic capabilities: the nature and microfoundations of (sustainable) enterprise performance," Strategic Management Journal, vol. 28, no. 13, pp. 1319-1350, 2007.

[13] H. Dzwigol, "The concept of the system approach of the enterprise restructuring process," Virtual Economics, vol. 2, no. 4, pp. 46-70, 2019.

[14] G. M. Jonathan, Influence of Organizational Structure on Business-IT Alignment: What We Do (Not) Know, Stockholm University, Kista, Swedan, 2018.

[15] J. M. Delaney and P. J. Devereux, "How gender and prior disadvantage predict performance in college," Economic and Social Review, vol. 51, no. 2, pp. 189-239, 2020.

[16] H. G. Barkema and M. Schijven, "Toward unlocking the full potential of acquisitions: the role of organizational restructuring," Academy of Management Journal, vol. 51, no. 4, pp. 696-722, 2008.

[17] M. J. Tippins and R. S. Sohi, "IT competency and firm performance: is organizational learning a missing link?" Strategic Management Journal, vol. 24, no. 8, pp. 745-761, 2003.

[18] K. Y. Lee and J. Kim, "Artificial intelligence technology trends and IBM Watson references in the medical field," Korean Medical Education Review, vol. 18, no. 2, pp. 51-57, 2016.

[19] J. Liu, H. Chang, J. Y.-L. Forrest, and B. Yang, "Influence of artificial intelligence on technological innovation: evidence from the panel data of China's manufacturing sectors," Technological Forecasting and Social Change, vol. 158, Article ID 120142, 2020.

[20] Y. Guo, X. Xia, S. Zhang, and D. Zhang, "Environmental regulation, government R\&D funding and green technology innovation: evidence from China provincial data," Sustainability, vol. 10, no. 4, p. 940, 2018.

[21] I. M. Cockburn, R. Henderson, and S. Stern, The Impact of Artificial Intelligence on Innovation, National Bureau of Economic Research, Cambridge, MA, USA, Retrieved from, 2018.

[22] S. Y. Lin, M. R. Mahoney, and C. A. Sinsky, "Ten ways artificial intelligence will transform primary care," Journal of General Internal Medicine, vol. 34, no. 8, pp. 1626-1630, 2019.

[23] M. F. Olmos and I. Díez-Vial, "Internationalization pathways and the performance of SMEs," European Journal of Marketing, vol. 49, no. 3/4, pp. 420-443, 2015.

[24] H. Chen, X. Li, S. Zeng, H. Ma, and H. Lin, "Does state capitalism matter in firm internationalization? pace, rhythm, location choice, and product diversity," Management Decision, vol. 54, no. 6, pp. 1320-1342, 2016.
[25] R. Cross, C. Ernst, D. Assimakopoulos, and D. Ranta, "Investing in boundary-spanning collaboration to drive efficiency and innovation," Organizational Dynamics, vol. 44, no. 3, pp. 204-216, 2015.

[26] A. Sandberg, "There is plenty of time at the bottom: the economics, risk and ethics of time compression," Foresight, vol. 21, no. 1, pp. 84-99, 2019.

[27] B. Cassiman and R. Veugelers, "In search of complementarity in innovation strategy: internal R\&D and external knowledge acquisition," Management Science, vol. 52, no. 1, pp. 68-82, 2006.

[28] Z. Liang and M. Song, "Time-consistent reinsurance and investment strategies for mean-variance insurer under partial information," Insurance: Mathematics and Economics, vol. 65, pp. 66-76, 2015.

[29] F. Rowe, D. Truex, and M. Q. Huynh, "An empirical study of determinants of e-commerce adoption in SMEs in Vietnam: an economy in transition," Journal of Global Information Management (JGIM), vol. 20, no. 3, pp. 23-54, 2012.

[30] N. Nan Zhang, X. Guo, G. Chen, and P. Y. K. Chau, "Impact of perceived fit on E-government user evaluation," Journal of Global Information Management, vol. 17, no. 1, pp. 49-69, 2009.

[31] K. H. Lim, "Knowledge management systems diffusion in Chinese enterprises: a multistage approach using the technology-organization-environment framework," Journal of Global Information Management (JGIM), vol. 17, no. 1, pp. 70-84, 2009.

[32] P. Antràs and D. Chor, "Organizing the global value chain," Econometrica, vol. 81, no. 6, pp. 2127-2204, 2013.

[33] T. Bassetti, Y. B. Galvez, F. Pavesi, and M. Del Sorbo, Artificial Intelligence: Impact on Total Factor Productivity, E-commerce \& Fintech:, JRC Technical report, Publications Office of the European Union, Luxembourg, 2020.

[34] N. Zhou and M. F. Guillén, "From home country to home base: a dynamic approach to the liability of foreignness," Strategic Management Journal, vol. 36, no. 6, pp. 907-917, 2015.

[35] L. P. Hansen, "Large sample properties of generalized method of moments estimators," Econometrica, vol. 50, no. 4, pp. 1029-1054, 1982. 